\title{
Ferramenta para Autoria e Acompanhamento de Atividades Metacognitivas no Ambiente Moodle
}

\author{
Eduardo da Silva Cruz, Edson Pinheiro Pimentel, \\ Juliana Cristina Braga, Wagner Tanaka Botelho \\ Universidade Federal do ABC (UFABC) \\ \{esilvac, edson.p.pimentel, jcbragal, wagtanaka\}@gmail.com
}

\begin{abstract}
Research has indicated that knowledge about one's own knowledge is essential to achieving quality learning. This knowledge can be enhanced with the development of metacognitive capacities, through activities that seek to involve the learner in monitoring their own knowledge. However, Virtual Learning Environments (AVA) such as Moodle, widely used by educators, do not natively have the tools to perform these activities, capture metrics, and analyze the results. This paper aims to present the MetaRef tool, a plug-in for Moodle, which allows organizing activities and collecting metacognitive metrics and also monitoring of these metrics by the student and the teacher.
\end{abstract}

Resumo. Pesquisas têm indicado que o conhecimento sobre o própria conhecimento é essencial para alcançar uma aprendizagem com qualidade. Este conhecimento pode ser realçado com o desenvolvimento de capacidades metacognitivas, por meio de atividades que buscam envolver o aprendiz no monitoramento do próprio conhecimento. No entanto, Ambientes Virtuais de Aprendizagem (AVA) como o Moodle, bastante utilizado por educadores, não possuem nativamente ferramentas que permitam realizar estas atividades, capturar métricas e analisar os resultados. Este artigo tem por objetivo apresentar a ferramenta MetaRef, um plug-in para o Moodle, que permite organizar atividades e coletar métricas metacognitivas e também o acompanhamento dessas métricas por parte do estudante e do professor.

\section{Introdução}

A Educação tem sido cada vez mais mediada por recursos tecnológicos, em menor ou maior grau, a depender da modalidade, presencial ou a distância. Ambientes Virtuais de Aprendizagem (AVA) como o Moodle [Moodle, 2019] têm sido utilizado no processo de ensino-aprendizagem para mediar os diversos elementos da relação entre estudante, professor e conteúdos. Pesquisas sobre o comportamento dos estudantes no AVA tem sido um tema recorrente [ROY; KUMAR, 2016], com diversas finalidades, como por exemplo, reduzir evasão [LEE; CHOI; KIM, 2013].

Pesquisas em Educação indicam que a aprendizagem efetiva acontece quando os aprendizes conseguem controlar a própria aprendizagem e se comprometem com o processo [FREIRE, 2009; DURALL e GROS, 2014, SETA, 2015; MOOLMAN, 2017]. O conhecimento sobre o próprio conhecimento é possível através do monitoramento da aprendizagem que é um dos aspectos da Metacognição, que em linhas gerais, constitui a 
VIII Congresso Brasileiro de Informática na Educação (CBIE 2019)

Anais do XXX Simpósio Brasileiro de Informática na Educação (SBIE 2019)

habilidade das pessoas em monitorar, avaliar, planejar ou controlar a própria aprendizagem [FLAVELL, 1979].

Este conhecimento metacognitivo pode ser realçado com o desenvolvimento de capacidades metacognitivas, por meio de atividades que buscam envolver o aprendiz no monitoramento do próprio conhecimento. De fato, diversos trabalhos têm apresentado protótipos ou ferramentas para, de alguma forma, propiciar a realização de atividades metacognitivas, mesmo que em domínios específicos.

No entanto, Ambientes Virtuais de Aprendizagem (AVA) como o Moodle, bastante utilizado por educadores, não possuem nativamente ferramentas que permitam realizar estas atividades metacognitivas, capturar métricas e analisar os resultados. No Moodle recursos adicionais podem ser implementados através da instalação e execução de recursos chamados plugins, que são extensões das funcionalidades apresentadas no ambiente Moodle padrão.

Realizou-se uma pesquisa preliminar em bases científicas por relatos sobre uso de plugins Moodle para fins de autoregulação da aprendizagem e o resultado retornou apenas a ferramenta PeerLA [KONERT et al (2016)]. No entanto, a ferramenta não consta na base oficial de plugins Moodle (https://moodle.org/plugins/) dificultando o seu uso. Em contato com os autores identificou-se que a ferramenta foi descontinuada por falta de recursos para continuidade da pesquisa.

Diante desse cenário, optou-se pelo desenvolvimento de uma ferramenta (plugin Moodle) que possa ser disponibilizada para a comunidade. Espera-se que a ferramenta crie condições para que mais educadores possam fazer uso de atividades que propiciem o desenvolvimento do conhecimento metacognitivo, coletando dados, analisando e aprimorando processos de ensino-aprendizagem.

Este artigo tem por objetivo apresentar a ferramenta MetaRef, um plug-in para o Moodle, que permite organizar atividades e coletar métricas metacognitivas. A ferramenta permite ainda o acompanhamento dessas métricas por parte do estudante e do professor.

O artigo está organizado como segue: na seção 2 são apresentados os fundamentos conceituais do trabalho e os trabalhos relacionados; a ferramenta MetaRef é descrita na seção 3, abordando sua arquitetura e funcionamento. Finalmente as conclusões e encaminhamentos de trabalhos futuros são apresentados na seção 4.

\section{Revisão de Literatura}

Esta seção apresenta os fundamentos conceituais necessários para a compreensão do trabalho. A partir de uma revisão de literatura conceituam-se os seguintes elementos: Autoregulação e Metacognição, e Métricas Metacognitivas. Na sequência alguns trabalhos relacionados são apresentados e discutidos.

\subsection{Autoregulação e Metacognição}

A autoregulação (self-regulation learning) é um aspecto importante do aprendizado do estudante e da performance acadêmica em sala de aula. De acordo com Pintrisch (1999), ela pode ser definida como estratégias que os estudantes utilizam para regular sua cognição assim como o uso de estratégias de gestão de recursos que os estudantes 
VIII Congresso Brasileiro de Informática na Educação (CBIE 2019)

Anais do XXX Simpósio Brasileiro de Informática na Educação (SBIE 2019)

utilizam para controlar seu aprendizado. Perrenoud (1999) define a regulação dos processos de aprendizagem como o conjunto das operações metacognitivas do sujeito e de suas interações com o meio que modificam seus processos de aprendizagem.

De acordo com Flavell (1979) essas operações metacognitivas referem-se à capacidade das pessoas em predizer seu desempenho sobre várias tarefas, isto é, quão bem elas serão capazes de lembrar vários estímulos e monitorar seu nível atual de perícia e compreensão. A metacognição é a capacidade do ser humano de monitorar e autoregular os processos cognitivos

Alhazmi (2017, p.18) apresenta o seguinte exemplo para diferenciar metacognição e cognição: “(...) programadores novatos sabem sobre habilidades cognitivas básicas, como constantes e variáveis; comparação e operadores lógicos; declarações de seleção; declarações iterativas; e matrizes. Quando eles precisam saber quando e como usá-los, isso é considerado uma habilidade metacognitiva. Segundo Gama (2005) as estratégias cognitivas ajudam os aprendizes a alcançar um objetivo, ao passo que as estratégias metacognitivas asseguram o alcance de um objetivo.

Zimmerman (2009) desenvolveu um modelo cíclico que chamou de fases do processo de autoregulação da aprendizagem. A figura 1 mostra, de forma adaptada, a versão atual do modelo de fases: a fase 1 lida com as estratégias e motivações; na fase 2 avaliam-se as capacidades de de autocontrole e autoobservação na realização de atividades; e na fase 3 o estudante realiza a autoreflexão sobre seus resultados.

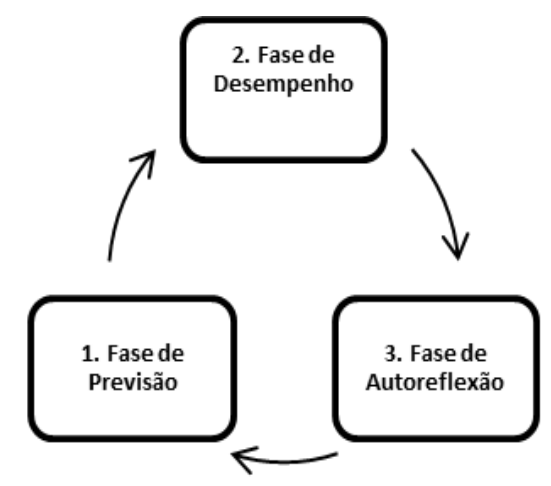

Figura 1. Fases do Processo de Autoregulação da Aprendizagem. Adaptado de Zimmerman e Moylan (2009)

\subsection{Métricas Metacognitivas}

A autoregulação pressupõe o uso de métricas que possam apoiar a identificação de ações regulatórias a partir das medidas obtidas, normalmente através de processos de avaliação da aprendizagem. Neste trabalho vamos utilizar as seguintes métricas, que serão detalhadas a seguir: KMA, KMB, SAA e SAB. As formulas para o cálculo dessas métricas podem ser encontradas nas referências de cada uma delas.

\subsubsection{KMA e KMB}

O KMA - Knowledge Monitoring Accuracy (precisão no monitoramento do conhecimento) foi definido por Tobias e Everson, (2002) e mede a diferença entre a estimativa do aluno sobre seu conhecimento antes de realizar uma avaliação e seu nível de conhecimentos determinado pelo seu desempenho na avaliação. 
VIII Congresso Brasileiro de Informática na Educação (CBIE 2019)

Anais do XXX Simpósio Brasileiro de Informática na Educação (SBIE 2019)

O valor do KMA está limitado no intervalo de -1.00 a 1.00 sendo que para valores compreendidos no intervalo de -0.25 a -1.00 o KMA é considerado Baixo, ou seja, o aprendiz não estima corretamente seu conhecimento na maioria das situações. Para valores compreendidos entre 0.50 e -0.25 , o KMA é Médio, ou seja, algumas vezes o aprendiz estima corretamente seu conhecimento, mas frequentemente comete erros médios ou grandes nas estimativas. E para valores compreendidos entre 1.00 e $0.50 \mathrm{o}$ KMA é Alto significando que na maioria das vezes o aprendiz estima corretamente o seu conhecimento.

O KMB - Knowledge Monitoring Bias (viés no monitoramento do conhecimento) foi definido por Gama (2005) e mede e identifica o tipo de desvio do aprendiz, sendo possível saber se o aluno é pessimista, aleatório ou otimista. Este tipo de informação permite uma precisão mais realista no monitoramento da aprendizagem e possibilita ao professor dar feedback mais adequado para cada aluno. Para o cálculo do KMB utilizam-se as mesmas variáveis descritas para o KMA.

O cálculo do KMB está relacionado ao do KMA e a faixa de valores também varia entre 1.00 e -1.00 . No entanto, os valores do KMB demonstram um significado diferente do KMA. Para os valores compreendidos no intervalo de 0.25 a 1.00 o aprendiz é considerado Otimista, pois tende a estimar que pode resolver o problema mas não o faz na maioria das situações. Os valores compreendidos entre 0.25 e -0.25 o aprendiz estima seu conhecimento ora de maneira otimista, ora de maneira pessimista, sendo considerado Aleatório. O aluno será considerado Pessimista, ou seja, tende a estimar que não pode resolver o problema mas o faz com sucesso em muitas situações, quando o valor do KMB estiver compreendido entre -1.00 e -0.25 . O aluno é considerado Realista quando o KMA for alto.

\subsubsection{SAA e SAB}

O SAA - Solution Analysis Accuracy (precisão na análise da solução) foi definido por (Pimentel, 2006) e é similar ao KMA. No entanto, essa métrica reflete a a precisão da comparação, pelo aprendiz, da sua solução com a solução do professor.

O SAB - Solution Analysis Bias (viés na análise da solução) foi definido também por (Pimentel, 2006) e é similar ao KMB. No entanto, essa métrica reflete o viés da comparação, pelo aprendiz, da sua solução com a solução do professor.

\subsection{Trabalhos Relacionados}

O principal trabalho relacionado é o de Konert et al (2016) que apresenta a ferramenta PeerLa, implementado como um conjunto de plugins para o Moodle v2.8, AVA amplamente utilizado nas universidades europeias, de acordo com os autores.

No PeerLA o material do curso deve ser pré-estruturado em seções e habilidades a serem cobertas dentro de um período de tempo restrito. Os aprendizes, na etapa de planejamento formulam metas, estimam o seu conhecimento prévio para cada uma das habilidades e informam os dias de estudo. No final de um "intervalo de aprendizagem" a ferramenta exibe aos alunos uma visão geral de suas metas e estes podem refletir sobre os aspectos resultados até o momento. Ao final do curso a ferramenta exibe a evolução e outros resultados na forma de gráficos. Os resultados apresentados em Konert et al (2016) indicam que o PeerLA é uma abordagem de sucesso para autoregulação da 
VIII Congresso Brasileiro de Informática na Educação (CBIE 2019)

Anais do XXX Simpósio Brasileiro de Informática na Educação (SBIE 2019)

aprendizagem. Em virtude da versão do plugin (para o Moodle v2.8) não foi possível testá-lo com versões atuais do Moodle.

Lima e Pimentel (2013) apresentam a ferramenta SRS - Self Regulation System que incorpora mecanismos para autoregulação da aprendizagem, utilizando como métricas o KMA e KMB. A ferramenta funcionava como um ambiente de avaliação da aprendizagem de maneira isolada, ou seja, não integrada a um AVA.

Kautzmann e Jaques (2016) propuseram um treinamento adaptativo específico da habilidade de monitoramento do conhecimento em sistemas tutores inteligentes e também fizeram uso da métrica KMA. Um agente pedagógico animado que realiza o treinamento proposto foi implementado e integrado a um sistema tutor algébrico. Por ser um STI a ferramenta é específica e não foi integrado a um AVA.

\section{A Ferramenta MetaRef}

Essa seção apresenta a ferramenta MetaRef - Metacognitive Reflection, planejada e desenvolvida como um plug-in Moodle, incorporando elementos para propiciar a autoregulação da aprendizagem. A Figura 2 apresenta o ciclo de aprendizagem adotado na ferramenta e inspira-se nas fases do Processo de Autoregulação da Aprendizagem. Adaptado de Zimmerman e Moylan (2009). A fase de autoreflexão (fase 3) de Zimmerman é desmembrada em 3 etapas na figura 2 (etapas 3, 4, e 5).

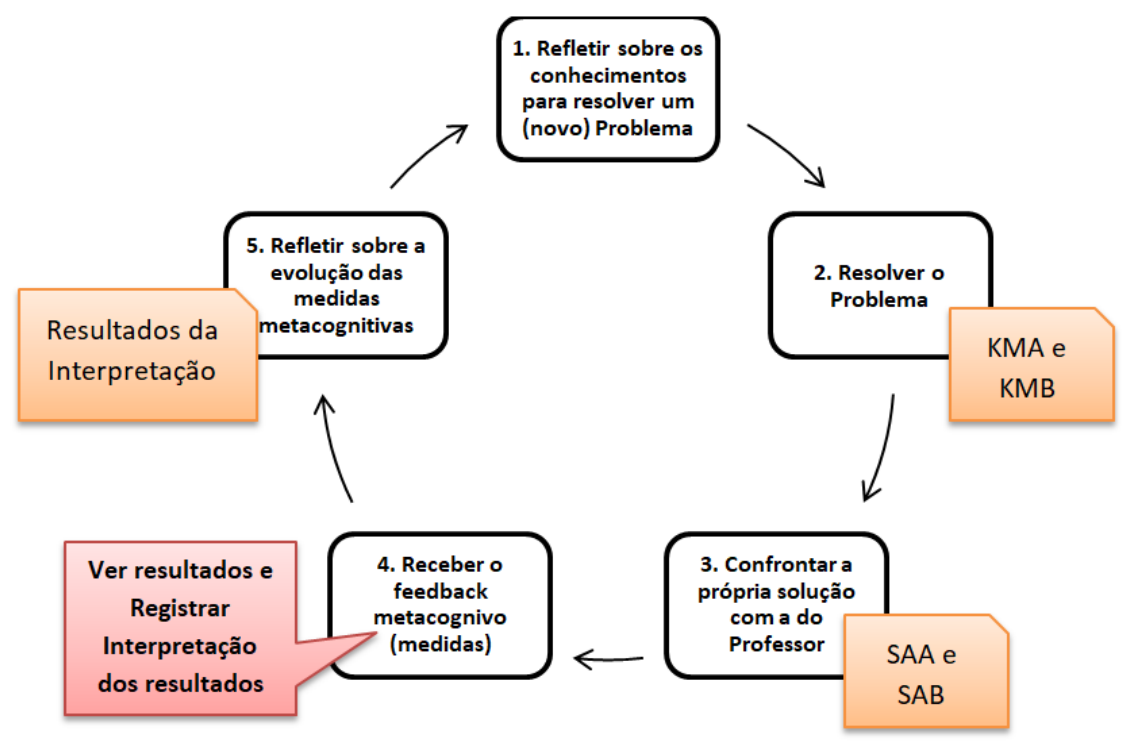

Figura 2. Ciclo de Aprendizagem na ferramenta MetaRef

As funcionalidades da ferramenta, no papel de professor e aluno são apresentadas na sequência. Um requisito estabelecido a priori foi que, para fins de reuso e aproveitamento, as atividades avaliativas seriam realizadas por meio dos recursos já disponíveis no Moodle como pesquisa (feedback), tarefa (task) e questionário (quiz).

\subsection{Configuração da Ferramenta pelo Professor}

Nesse trabalho denominamos de "Seção Metacognitiva" (SM) o conjunto de etapas necessárias para cumprir o fluxo Previsão-Desempenho-Reflexão. Para cada SM o professor deverá realizar a seguinte sequência de passos: 
VIII Congresso Brasileiro de Informática na Educação (CBIE 2019)

Anais do XXX Simpósio Brasileiro de Informática na Educação (SBIE 2019)

I. Adicionar um recurso do tipo "Pesquisa" para a previsão de desempenho na solução de um problema;

II. Adicionar um recurso do tipo "Questionário" ou "Tarefa" para a resolução do respetivo problema;

III. Adicionar um recurso do tipo "MetaRef" e configurá-lo, associando os respectivos recursos criados nos passos I e II.

No passo "I" a previsão de desempenho deverá ser realizada descrevendo-se o enunciado de um problema e solicitando que se responda a uma "Pesquisa" conforme figura 3.

\section{3-13A-Estrutura de Repetição - PREVISÃO DE DESEMPENHO}

Após refletir sobre as perguntas/sentenças

apresentadas faça uma previsão do seu ALTO: pensa que pode responder completamente (mais de 75\%).

GRAU DE ACERTO ao respondê-las.(C)

MÉDIO: pensa que pode responder apenas parcialmente (entre 50\% e 75\%)

BAIXO: pensa que pode responder menos de $50 \%$

\section{Figura 3. Recurso Pesquisa do Moodle - Previsão de Desempenho}

No passo "II" o problema a ser resolvido deve ser criado com o mesmo enunciado da previsão na forma de "questionário" (múltipla escolha) ou tarefa dissertativa. Deve-se utilizar a opção "Restringir Acesso" (figura 4), de modo que o estudante somente possa resolver o problema após fazer a previsão de desempenho da respectiva atividade.

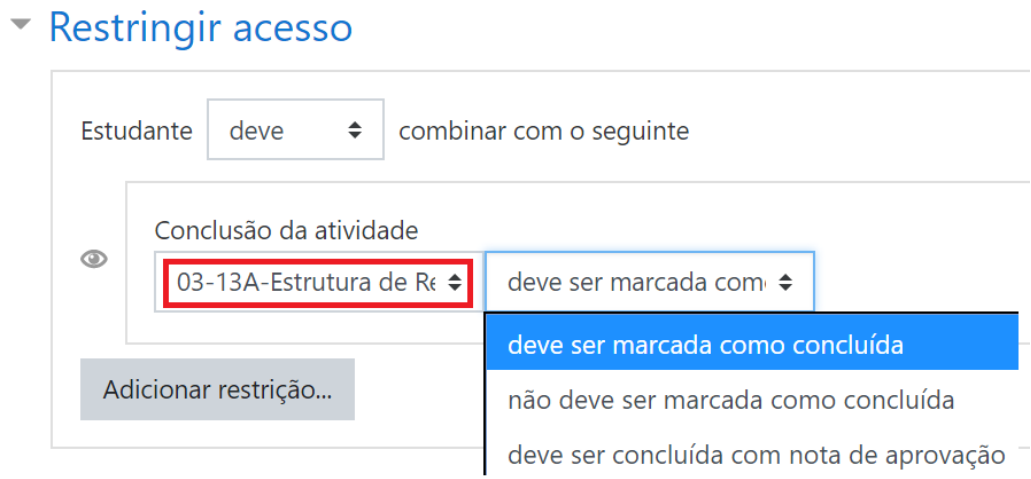

Figura 4. Restringir Acesso ao Recurso de Realização de Desempenho.

No passo "III" a configuração do MetaRef deve ser feita conforme figura 5, mediante os seguintes passos:

[1] Habilitar ou não as métricas KMA/KMB;

[2] Selecionar a Atividade de Previsão de Desempenho (Pesquisa);

[3] Indicar se a tarefa de desempenho será uma atividade ou quiz e selecionar a respectiva tarefa;

[4] Indicar se a resposta deve ou não ser mostrada na etapa de Reflexão. 
VIII Congresso Brasileiro de Informática na Educação (CBIE 2019)

Anais do XXX Simpósio Brasileiro de Informática na Educação (SBIE 2019)

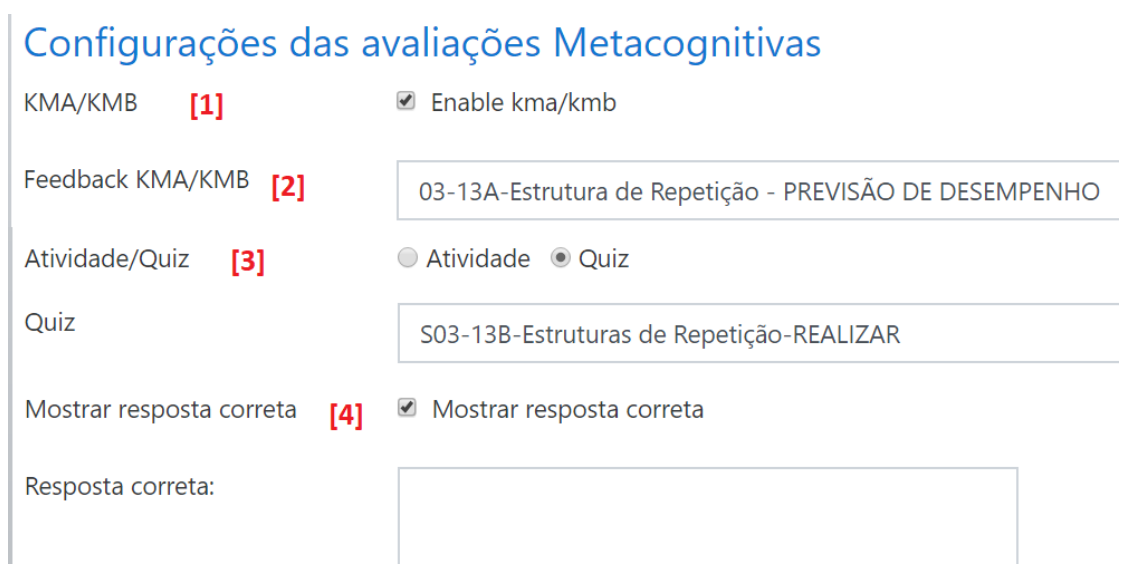

Figura 5. Configuração do Recurso MetaRef.

\subsection{Uso da Ferramenta pelo Aprendiz}

Cada SM dever ser realizada pelo estudante no prazo definido nas respectivas atividades. Após realizar a previsão (passo I) e o desempenho (passo II) estudante consegue acessar o recurso MetaRef para visualização de resultados e reflexão guiada.

Na etapa de reflexão o primeiro passo é a ANÁLISE DA SOLUÇÃO, conforme figura 6. O estudante deve, para a questão apresentada no enunciado do problema, confrontar suas respostas e as respostas corretas, selecionando os itens "As questões", "Suas Respostas" e "Respostas Corretas". Após suas análises deve, no item "Reflexão sobre a solução" indicar o quão próxima sua solução está da solução das opções, assinalando uma das três opções. Ao clicar em "Salvar Mudanças" a tela seguinte será apresentada (figura 7).

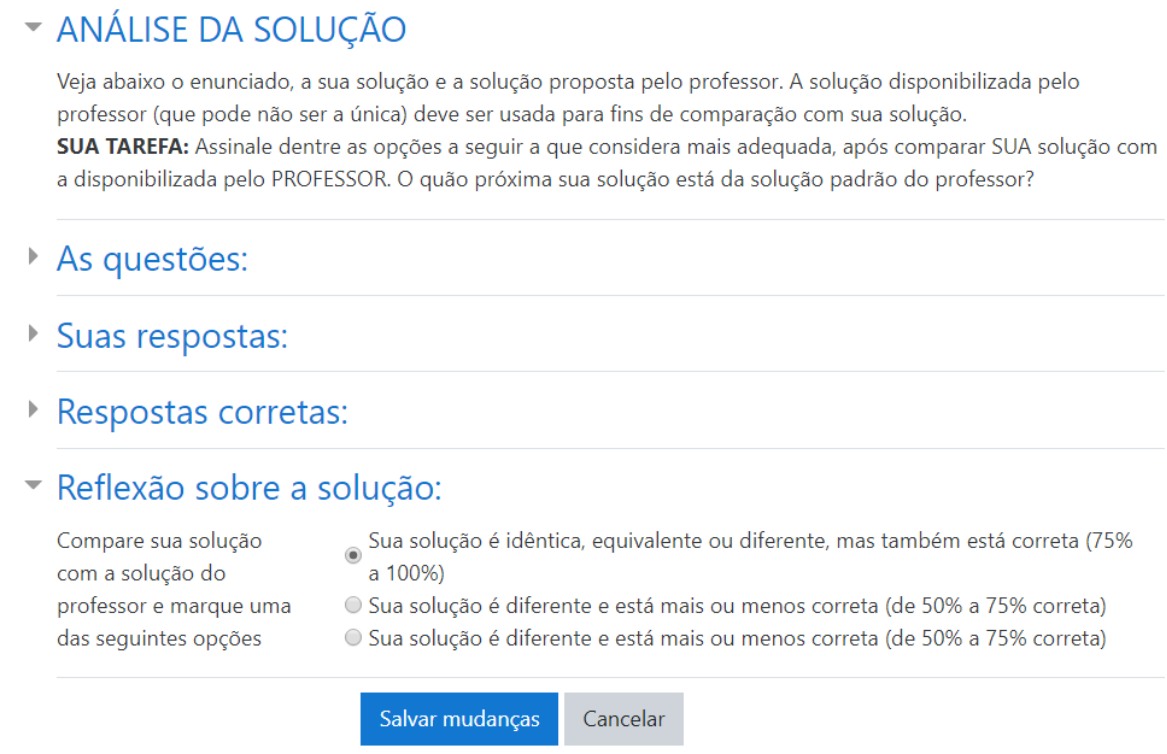

Figura 6. Análise da Solução pelo Estudante.

$\mathrm{Na}$ tela mostrada na figura 7, o estudante consegue visualizar os históricos de suas previsões e desempenhos, as diferenças, e o seu KMA e KMB atual. Ao clicar no item "Tabela" (figura 7) o estudante consegue ver a interpretação do KMA e KMB, para cada faixa de valores (não exibida nesse artigo por questões de espaço). 
VIII Congresso Brasileiro de Informática na Educação (CBIE 2019)

Anais do XXX Simpósio Brasileiro de Informática na Educação (SBIE 2019)

Resultados Gerais Metacognitivos

Os dados a seguir mostram seus resultados históricos de PREVISÃo de desempenho e EFETIVO desempenho em problemas anteriores. Olhe para seus dados, especialmente as colunas "DIFERENÇAS" (na pré e pós avaliação) e reflita sobre seus avanços.

\begin{tabular}{|c|c|c|c|c|c|}
\hline Atividade/Quiz & $\begin{array}{c}\text { Seu } \\
\text { Resultado }\end{array}$ & $\begin{array}{c}\text { Sua Pré- } \\
\text { Avaliação }\end{array}$ & $\begin{array}{c}\text { Diferença na Pré- } \\
\text { Avaliação }\end{array}$ & $\begin{array}{c}\text { Sua Pós } \\
\text { Avaliação }\end{array}$ & $\begin{array}{c}\text { Diferença Pós } \\
\text { Avaliação }\end{array}$ \\
\hline $\begin{array}{c}\text { 01-01B-Introdução à Programação de } \\
\text { Computadores - RESPONDER }\end{array}$ & Alta & Alta & $0 \%$ & Alta & $0 \%$ \\
\hline 01-02B-Algoritmos Computacionais - RESPONDER & Alta & Alta & $0 \%$ & Alta & $0 \%$ \\
\hline 01-03B-Algoritmos Sequenciais-RESPONDER & Baixa & Média & $50 \%$ & Média & $50 \%$ \\
\hline $\begin{array}{c}\text { 02-06B-Estrutura de Seleção Simples e Composta - } \\
\text { RESPONDER }\end{array}$ & Média & Média & $0 \%$ & Média & $0 \%$ \\
\hline $\begin{array}{c}\text { 02-07B-Estrutura de Seleção Encadeada - } \\
\text { RESPONDER }\end{array}$ & Alta & Média & $50 \%$ & Média & $50 \%$ \\
\hline 02-08B-Estrutura de Seleção - RESPONDER & Média & Média & $0 \%$ & Média & $0 \%$ \\
\hline $\begin{array}{c}\text { S03-11B-Estrutura de Repeticão Enquanto - } \\
\text { REALIZAR }\end{array}$ & Alta & Média & $50 \%$ & Média & $50 \%$ \\
\hline S03-13B-Estruturas de Repetição-REALIZAR & Alta & Média & $50 \%$ & Média & $50 \%$ \\
\hline
\end{tabular}

Seu KMA é: 0.25000 . Seu KMB é: -0.12500

Seu SAA é: $\mathbf{0 . 8 1 2 5 0}$. Seu SAB é: 0.06250

$\checkmark$ Tabela

Figura 7. Resultados Gerais Metacognitivos.

Na sequência o estudante deverá dialogar com os seus resultados, assinalando as opções de resposta conforme a figura 8. Esse diálogo tem por finalidade verificar se o estudante está compreendendo os seus resultados metacognitivos.

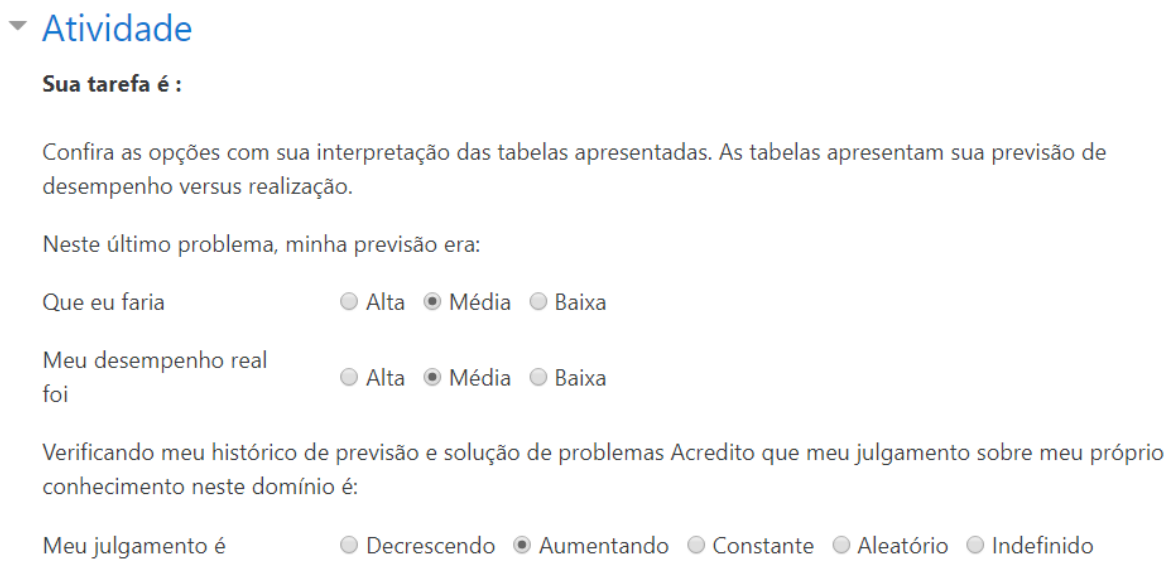

\section{Salvar mudanças Cancelar}

Figura 8. Interpretação dos Resultados Metacognitivos.

Por fim, a ferramenta confronta a interpretação do estudante com os registros e informa como está sua compreensão (baixa, media, alta) e explica como está sendo sua tendência, conforme figura 9. Além disso, convida o estudante a se lembrar desse feedback na realização da próxima atividade.

\subsection{Análise de Resultados Metacognitivos pelo Professor}

Ao acessar o recurso MetaRef em cada SM o professor tem acesso aos resultados de todos os estudantes, naquela SM e também aos resultados históricos (de todas as SM) conforme mostra a figura 10. Note, por exemplo, que o estudante com id $=18$, obteve $\mathrm{KMA}=-0.50$ na SM “03-13” (figura 10a) e seu KMA geral 0.25 (figura 10b). 
VIII Congresso Brasileiro de Informática na Educação (CBIE 2019)

Anais do XXX Simpósio Brasileiro de Informática na Educação (SBIE 2019)

\title{
ANÁLISE DO ESTADO ATUAL DE MONITORAMENTO PRÓPRIO CONHECIMENTO
}

\begin{abstract}
Como está sua precisão em estimar seu conhecimento (metacognição)?
Você demonstrou Baixa precisão em julgar seus conhecimentos neste curso. Tenha em mente que esta é apenas uma análise aproximada, isso sugere que você é Você demonstrou não entende bem o que você "sabe" e "não sabe". Verifique o feedback em sua solução e tente identificar suas lacunas de aprendizado.
\end{abstract}

Como está sua tendência em estimar seu conhecimento? Você está sendo otimista ou pessimista?

Em média, você está sendo Pessimista ha sua avaliação do seu conhecimento, suas habilidades na resolução de problemas. Isso significa que você imagina que você sabe MAIS do que você demonstra.

Veja abaixo o seu KMA e o seu KMB médio:

Seu KMA é: $\mathbf{0 . 2 5 0 0 0}$. Seu KMB é: $-\mathbf{0 . 1 2 5 0 0}$

Seu SAA é: $\mathbf{0 . 8 1 2 5 0}$. Seu SAB é: $\mathbf{0 . 0 6 2 5 0}$

Figura 9. Resultado da Interpretação Metacognitiva pelo Estudante.

\section{Considerações Finais}

Esse trabalho apresentou a arquitetura e o funcionamento da ferramenta MetaRef, um plugin para o AVA Moodle, bastante utilizado pelos educadores. A ferramenta implementa parcialmente o ciclo de autoregulação da aprendizagem de Zimmerman.

Os dados que são coletados e apresentados por meio da ferramenta propiciam ao estudante o desenvolvimento do conhecimento metacognitivo, uma vez que realiza o díalogo reflexivo sobre os resultados e interpretações. Os dados apresentados na figura são de um experimento em andamento em uma disciplina de graduação.

Os próximos passos desse trabalho incluem validar a ferramenta, inclusive com grupos de controle, de modo a verificar se as sessões metacognitivas, como propostas, de fato melhoram o conhecimento metacognitivo. Além disso, pretende-se verificar se a melhoria do conhecimento metacognitivo também impacta melhora os resultados de aprendizagem. Como trabalhos futuros, planeja se incrementar a ferramenta com mais elementos do ciclo de autoregulação proposto por Zimmerman.

03-13C-Estrutura de Repetição - META

\begin{tabular}{|c|c|c|c|c|c|}
\hline Userid & Username & KMA & SAA & KMB & SAB \\
\hline 18 & -0.5 & 1.0 & -0.5 & 0.0 \\
\hline 68 & -0.5 & 1.0 & 0.5 & 0.0 \\
\hline 106 & 1.0 & -0.5 & 0.0 & 0.5 \\
\hline 43 & -0.5 & -0.5 & 0.5 & 0.5 \\
\hline 45 & 1.0 & 1.0 & 0.0 & 0.0 \\
\hline 105 & jannafic & 1.0 & 1.0 & 0.0 & 0.0 \\
\hline Average & & 0.25000 & 0.50000 & 0.08333 & 0.16667 \\
\hline
\end{tabular}

(a) Resultados da SM

General Metacognition Results

\begin{tabular}{|c|c|c|c|c|c|}
\hline Userid & Username & KMA & SAA & KMB & SAB \\
\hline 18 & & 0.25000 & 0.81250 & -0.12500 & 0.06250 \\
\hline 68 & & 0.62500 & 1.00000 & 0.12500 & 0.00000 \\
\hline 106 & 0.11111 & 0.33333 & 0.11111 & 0.22222 \\
\hline 43 & & -0.16667 & 0.33333 & 0.05556 & 0.22222 \\
\hline 45 & 1.00000 & 1.00000 & 0.00000 & 0.00000 \\
\hline 105 & & 0.57143 & 0.78571 & 0.00000 & 0.07143 \\
\hline
\end{tabular}

(b) Resultados Gerais

Figura 9. Resultado da Interpretação Metacognitiva pelo Estudante.

\section{Referências}

ALHAZMI, Ahoud. Providing Metacognitive Support Using Learning by Teaching Paradigm. 2017. University of Adelaide. Dissertação de Mestrado. 
VIII Congresso Brasileiro de Informática na Educação (CBIE 2019)

Anais do XXX Simpósio Brasileiro de Informática na Educação (SBIE 2019)

DURALL, E.; GROS, B. Learning Analytics as a Metacognitive Tool. Proceedings of the 6th International Conference on Computer Supported Education-Volume 1. Anais...SCITEPRESS-Science and Technology Publications, Lda, 2014

FLAVELL, J. H. (1979) Metacognition and cognitive monitoring: A new area of cognitive-developmental inquiry. American Psychologist, 34, 906-911.

FREIRE, L. G. L. Auto-regulação da aprendizagem. Ciências \& Cognição, v. 14, n. 2, p. 276-286, 2009.

GAMA, Claudia A. Integrating metacognition instruction in interactive learning environments. University of Sussex, 2005. Tese de Doutorado.

KAUTZMANN, Tiago Roberto; JAQUES, Patrícia A. Treinamento da Habilidade Metacognitiva de Monitoramento do Conhecimento em Sistemas Tutores. Revista Brasileira de Informática na Educação, v. 24, n. 2, 2016.

LEE, Y.; CHOI, J.; KIM, T. Discriminating factors between completers of and dropouts from online learning courses. British Journal of Educational Technology, v. 44, n. 2 , p. 328-337, 2013.

LIMA, Allen F. Oberleitner; PIMENTEL, Edson P. Mecanismos para Suporte à AutoRegulação da Aprendizagem do Estudante. In: Brazilian Symposium on Computers in Education (Simpósio Brasileiro de Informática na Educação-SBIE). 2013. p. 296.

MOODLE, Disponível em https://moodle.org/. Acesso em 07 de Julho de 2019.

PERRENOUD, Philippe. Avaliação: da excelência à regulação das aprendizagens entre duas lógicas. Porto Alegre; Artmed, 1999.

PIMENTEL, E. P. (2006). Um modelo para avaliação e acompanhamento contínuo do nível de aquisição de conhecimentos do aprendiz. Instituto Tecnológico de Aeronáutica (ITA), Brasil. Tese de Doutorado.

PINTRICH, P. R. The role of motivation in promoting and sustaining self- regulated learning. International Journal of Educational Research, v. 31, p. 459-470, 1999.

ROY, M.; KUMAR, R. Informing Authoring Best Practices Through an Analysis of Pedagogical Content and Student Behavior. (A. Micarelli, J. Stamper, K. Panourgia, Eds.) Intelligent Tutoring Systems.: Lecture Notes in Computer Science. Springer International Publishing, 2016

SETA, Kazuhisa; TANIGUCHI, Yuki; IKEDA, Mitsuru. Learner Modeling to Capture Meta-Cognitive Activities through Presentation Design. the Journal of Information and Systems in Education, v. 14, n. 1, p. 1-12, 2015.

ZIMMERMAN, Barry J.; MOYLAN, Adam R. Self-regulation: Where metacognition and motivation intersect. In: Handbook of metacognition in education. Routledge, 2009. p. 311-328.

MOOLMAN, Ruan. Metacognitive skills of second year extended and main stream University mathematics students: a case study. 2017. University of the Witwatersrand, Johannesburg Tese de Doutorado. 